\title{
OBSTETRICIA EN ADOLESCENTES
}

\section{Dr. Jesús Alberto Gómez Palacino*}

Puesto que no existe en la literatura médica nacional y es muy escasa la bibliografía del mundo acerca de lo que ocurre en la paciente obstétrica joven, consideramos de interés analizar este capítulo subestimado de la especialidad gineco-obstétrica.

Es curioso que mientras se concede gran trascendencia a otros aspectos de la reproducción humana, se ignore la problemática de las adolescentes. Ello es más sorprendente aún, cuando nos enfrentamos en la actualidad a circunstancias nuevas que hacen imperioso el conocimiento de fenómenos sociológicos vigentes.

Si tenemos en cuenta que según el censo de Estados Unidos de Norte América de 1960, citado por Castellanos y cols. (5) el $42,5 \%$ de los matrimonios entre blancos y el $57,5 \%$ de negros se efectuaron entre jóvenes, y de las novias la tercera parte o la mitad estaban embarazadas en el momento de contraer matrimonio y según Claman y Bell (6), en su investigación en la Columbia Británica, en los últimos diez años el número de pacientes adolescentes se ha triplicado, es de suponer que un gran núcleo humano ha escapado del análisis.

Si aceptamos con Goodrich Schauffler (14) que la adolescencia es el período que se inicia un año antes de la menarquia y termina con la madu- rez, tenemos que admitir que la gestación, durante esta etapa de desarro1lo, debe tener aspectos peculiares que nos proponemos estudiar.

\section{Material y Métodos}

Se revisaron 600 historias clínicas de pacientes de 13, 14 y 15 años atendidas en el Instituto Materno Infantil de Bogotá en el período comprendido entre 1963 y 1967. En el mismo lapso de tiempo se hospitalizaron 25.257 pacientes primigestantes lo cual significa que el 2,37\% correspondió a las adolescentes de nuestro estudio, casi todas también primigestantes.

Con el objeto de poder establecer comparaciones y obtener conclusiones tomamos un grupo de referencia integrado por pacientes de 20 años.

La distribución e incidencia por edades, el estado civil y su asistencia a la consulta prenatal se expresan en el siguiente cuadro:

\section{CUADRO № 1}

\begin{tabular}{ccccc}
\hline Edad & Casos & Solteras & Casadas & Prenatal \\
\hline 13 & 25 & $80 \%$ & $20 \%$ & $12 \%$ \\
14 & 97 & $80 \%$ & $20 \%$ & $11,3 \%$ \\
15 & 478 & $61 \%$ & $39 \%$ & $17,9 \%$ \\
\hline
\end{tabular}

* Profesor Asociado de Obstetricia y Ginecología. Facultad de Medicina - Universidad Nacional. 
Como se observa, el porcentaje de madre-solterismo es muy elevado y probablemente ha de tener influencia en algunos aspectos de la patología obstétrica de la adolescente.

La asistencia a la consulta prenatal fue muy pobre como que solamente lo hizo la sexta parte del total de las pacientes y muchas de ellas de manera insuficiente pues concurrieron tan solo una o dos veces. Aun cuando este fenómeno es destacado por algunos autores, no le concedemos mayor trascendencia para nuestro grupo en estudio porque también se presentó en otros de mayor edad. Sin embargo se destaca cómo a medida que la edad fue en aumento la asistencia al control se incrementó como que el mejor nivel de maduración psicológica facilitó la búsqueda del examen médico. Esta circunstancia además se confirmó con una mejor motivación entre casadas que entre solteras.

\section{Evolución del Embarazo.}

\section{Toxemia}

Hubo entre las adolescentes 116 casos de Toxemia, lo cual significó una incidencia de 19,3\%. El siguiente cuadro muestra datos estadísticos de diversos autores que permiten comparar los nuestros:

CUADRO Nํ 2

INCIDENCIA DE TOXEMIA

EN ADOLESCENTES

Israel (10)

Bochner (22)

Sinclair (16)

Hofmeister (9)

Poliakoff (13)

PRESENTE ESTUDIO

Marchetti (.11)

Mussio (12)

Aznar (1)

Claman (6)

$6,0 \%$

$11,0 \%$

$11,0 \%$

$11,7 \%$

$17,7 \%$

$19,3 \%$

$20,0 \%$

$28,0 \%$

$29,8 \%$

$32,0 \%$

El siguiente Cuadro, № 3, que registra la evolución del embarazo, muestra cómo en nuestra casuística la incidencia de toxemia en el grupo de pacientes de 20 años fue también elevada, $17 \%$, lo cual sugiere que el solo hecho de la primigestación, independientemente de la edad, pudo predisponer a la toxemia.

\section{CUADRO N: 3}

EVOLUCION DEL EMBARAZO

\begin{tabular}{|c|c|c|c|c|c|}
\hline Edad & Aborto & P. Inmaduro & P. Prematuro & A Término & Toxemia \\
\hline 13 & $8 \%$ & & $20,0 \%$ & $72,0 \%$ & $16,0 \%$ \\
\hline 14 & $13 \%$ & $2,8 \%$ & $22,7 \%$ & $61,5 \%$ & $18,4 \%$ \\
\hline 15 & $5 \%$ & $1,6 \%$ & $15,6 \%$ & $77,0 \%$ & $19,2 \%$ \\
\hline 20 & $3 \%$ & & $11,0 \%$ & $86,0 \%$ & $17,0 \%$ \\
\hline
\end{tabular}

Hubo 10 casos de Eclampsia en adolescentes y su porcentaje, 1,66\%, resultó ser 10 veces mayor que el aportado por la estadística Internacional de Eclampsia, que según Doll y Hanington (7) oscila entre 2 y 1,6 por mil partos.

\section{Abortos, Partos Inmaduros y Prematuros}

44 pacientes abortaron correspondiendo el mayor número a adolescentes de 14 años. El porcentaje total de interrupciones precoces de la ges- 
tación alcanzó a 7,3\%, cifra superior al $3 \%$ de las pacientes del grupo control de 20 años.

4 abortos fueron provocados y de ellos uno en una adolescente de 14 años quien lo repetía en su segunda gestación.

En el estudio de Aznar y Lara (1) la incidencia de aborto en pacientes por debajo de 16 años llegó a 8,7\% $y$ en el de Semmens (15) en menos de $15,5,5 \%$.

5 partos fueron inmaduros al paso que en las pacientes de 20 años no los hubo.

Los índices de prematuridad fueron significativamente altos: $20,22,7$ y $15,6 \%$ respectivamente para adolescentes de 13,14 y 15 años en contraste con el $11 \%$ de las gestantes de 20 años.

Solamente llegaron al término del embarazo $74,3 \%$ de las adolescentes a diferencia de $86 \%$ de las del grupo control.

Hubo 3 molas hidatiformes, una grado I y dos de grado II de la Clasificación de Hertig, en pacientes de 13, 15 y 17 años respectivamente, lo cual significa que su incidencia fue 3 veces superior a la encontrada para la frecuencia general de la mola.

\section{Parto}

Hubo 556 partos. La terminación del embarazo se verificó así:

\section{CUADRO № 4}

PARTOS

\begin{tabular}{lrr}
\hline Parto espontáneo & 447 & $79,7 \%$ \\
Fórceps & 81 & $14,5 \%$ \\
Cesárea & 13 & $2,3 \%$ \\
Espátulas de Tierry & 9 & \\
Extracción podálica & 4 & \\
Ventosa eutósica & 2 & \\
\hline
\end{tabular}

Conviene advertir que la mayoría de las aplicaciones de fórceps tuvo indicación profiláctica y que los terapéuticos obedecieron a indicaciones diversas.

La intervención cesárea fue practicada por las siguientes indicaciones:

\section{CUADRO № 5}

CESAREAS - INDICACIONES

\begin{tabular}{lc}
\hline & Casos \\
\hline Sufrimiento fetal & 5 \\
Desproporción céfalo-pélvica & 3 \\
Distocias de encajamiento & 3 \\
Situación transversa & 1 \\
Procidencia del cordón & 1 \\
\hline
\end{tabular}

En el grupo de gestantes de 20 años la cesárea se practicó en el $3 \%$ de los casos. El parto se produjo en presentación cefálica en $96 \%$ de los casos y en presentación de pelvis en $3,9 \%$. Hubo una situación transversa.

\section{Duración del Trabajo}

El promedio de duración del trabajo fue de 9 horas 52 minutos mientras que en pacientes del grupo control fue de 8 horas, 45 minutos.

Aún cuando se advierte una mayor duración del trabajo se considera que evolucionó dentro de los términos normales y que salvo las distocias que impusieron la terminación artificial del parto, no hubo incidencias atribuíbles a la edad de las pacientes. Por otra parte su comportamiento tanto psíquico como físico durante el trabajo fue normal. En el estudio de Brochier y Rascle (4) sobre 220 casos de pacientes por debajo de 16 años encontró estrechez pélvica en el $12 \%$ de las adolescentes menores de 14 años, mientras que entre las de 14 a 15 años el porcentaje disminuyó a $5,5 \%$ y concluyeron que en Francia las niñas ad- 
quieren sus caracteres de nubilidad a los 15 años. Probablemente la mayor duración del trabajo de parto pueda estar relacionada con dificultades que la pelvis ósea materna, aun no desarrollada, pueda ofrecer.

\section{Puerperio}

Salvo 5 casos de sepsis moderadas (endometritis y mastitis) y 6 casos de dehiscencia de episiorrafias, no hubo otras complicaciones en la evolución del puerperio.

\section{Recién Nacidos - Resultados}

Habiendo ocurrido 3 partos de gemelos, el número posible de fetos vivos fue de 559 cuya distribución por pesos se establece así:

\section{CUADRO NN 6}

PESO DE LOS RECIEN NACIDOS

\begin{tabular}{llrr}
\hline De 501 a 999 & 5 & $0,7 \%$ \\
De 1.000 a 2.499 & 123 & $22,0 \%$ \\
De 2.500 o más & 431 & $77,1 \%$ \\
\hline
\end{tabular}

Obsérvese el alto número de recién nacidos de menos de 2.500 grs., por encima del dato aportado por Semmens (15) de 9,5\% y del Poliakoff (13) de $17,4 \%$ en pacientes de las mismas edades estudiadas por nosotros.

\section{Malformaciones congénitas}

Hubo 17 casos de fetos malformados por una incidencia de 3\%, ocurriendo la mayor parte entre adolescentes de 14 años en las cuales su frecuencia ascendió a 4,7\% al paso que en el grupo control la incidencia solamente llegó a 1\%. Criptorquidia, displasias del pie (equino-valgus, equino-varus, talus), luxación de la cadera, hidrocele, labio leporino y paladar hendido fueron las malformaciones encontradas. Hubo además un caso de Trisomía GI.
En la revisión de Briggs y cols. (3) se anotaron 3 casos de muertes neonatales en hijos de adolescentes, 2 de ellas por anomalías congénitas; en el estudio de Mussio (12) en pacientes de 12 a 14 años hubo $2 \%$ de anomalías congénitas y Glaman y Bell al informar sobre 224 casos de pacientes adolescentes anotó 8 mortinatos 3 de ellos con serias anomalías.

\section{Mortalidad fetal}

Teniendo en cuenta que hubo 13 partos con fetos mortinatos $(2,3 \%)$ y 17 muertes neonatales precoces (3\%), la mortalidad perinatal ascendió a $5,3 \%$ en contraste con el $1 \%$ del grupo de pacientes de 20 años.

Las muertes neonatales ocurrieron en la mayor parte de los casos por inmaciurez, prematurez y membrana hialina. Un feto con malformaciones múltiples falleció.

Hofmeister (9) reportó una mortalidad fetal en adolescentes de 12 a 15 años de $4,41 \%$ y Poliakoff (13) informó cifras muy semejantes a las nuestras: mortinatos $2,4 \%$, neonatales $3,5 \%$ y perinatal de $5,9 \%$.

\section{Comentarios y Conclusiones}

Los datos estadísticos presentados ponen de manifiesto que el embarazo en las pacientes adolescentes debe ser mirado con reservas en virtud de los siguientes hallazgos:

1. Mayor incidencia de abortos, partos inmaduros y partos prematuros.

2: Elevado porcentaje de toxemia gravídica.

3. Aumento en la frecuencia de malformaciones congénitas.

4: Mortalidad perinatal elevada. 
Queriendo dar explicación a la morbilidad expresada en las 4 conclusiones anteriores es preciso admitir la falta de desarrollo psicosomático en las adolescentes como su causa eficiente.

Fue notoria la deficiente o nula atención dispensada en el período prenatal y al respecto es lógico pensar que el escaso nivel cultural propio de mentes inmaduras hace aun más comprometedora la acción profiiáctica. Bernstein, citado por Claman y Bell (6), en su estudio sobre las fallas de los servicios sociales para madres solteras, señala que las largas esperas y hospitales llenos de gente, localizados lejos del hogar, la profusión de médicos y la falta de reserva impiden a las adolescentes, tímidas y atemorizadas, buscar la atención adecuada a su estado prenatal.

Para dar explicación a la elavada incidencia de toxemia en las adolescentes bastaría invocar: a) falta de desarrollo y desequilibrio endocrino, b) dieta inadecuada principalmente en el aporte protéico con el consiguiente daño hepático, c) oposición anatómica a la distención del músculo uterino, propia de las primigestantes, y por ende, liberación de substancias vasopresoras facilitada por la isquemia local y d) desequilibrio psicoemocional a expensas de situaciones de conflicto.

Es significativo el hallazgo de Poliakoff (13) quien encontró una incidencia de $37,7 \%$ de toxemia en pacientes jóvenes casadas contra $62,3 \%$ en las solteras. Los estudios de Glick y cols. (8) acerca de los factores psicofisiológicos en la génesis de la toxemia confirmaron la mayor frecuencia de la disgravidia en pacientes con antecedentes de niñez trastornada emocionalmente $y$ en solteras.
La inmadurez endocrina y la falta de desarrollo completo del útero justificarían, en parte, la precipitación del parto extemporáneamente.

Es de presumir que fallas en la etapa de gametogénesis, de segmentación y de embriogénesis en sistemas biológicos aun no completamente desarrollados faciliten la interrupción precoz de embarazos anormales y en el mismo sentido se podrían interpretar los accidentes malformativos, más frecuentes en las adolescentes que en las pacientes obstétricamente maduras.

El elevado porcentaje de niños de bajo peso, con todas sus consecuencias, y la frecuencia de toxemia gravídica con placentas funcionalmente deficientes explicaría la mortalidad perinatal.

En contraste con los fenómenos adversos explicados, la duración del trabajo del parto, la evolución del mismo y la baja frecuencia de nacimientos intervenidos permiten afrontar su atención sin sobresaltos pues los accidentes y complicaciones rara vez ocurren. Sin embargo el hecho de que los fetos sean con frecuencia de bajo peso impone los cuidados que el parto prematuro exige.

Quizá la facilidad del parto espontáneo y los resultados obtenidos en las adolescentes permitió a Harris, citado por Marchetti y Menaker (11) considerar que la edad de 16 años - menos fuese la apropiada para tener el primer hijo, criterio discutible que no fue confirmado por nosotros.

Nuestro estudio señala la necesidad de estimular la educación sexual y los conocimientos acerca de la reproducción humana en edad temprana. Quizá debería incluirse en los programas escolares y desde luego incrementarse en el propio hogar. Dado 
que muchas adolescentes están aún en manos de los médicos pediatras sería deseable que ellos también asumieran funciones en la enseñanza sexual.

Respecto a ginecólogos y obstetras, la comprensión y apoyo psicológicos deben ser tan fundamentales como los mismos procedimientos tocúrgicos con miras a obtener los mejores resultados tanto maternos como fetales en la atención de las adolescentes.

\section{BIBLIOGRAFIA}

1 AZNAR, R., y LARA, R.: Gin. y Obs. de México. XXII: $661 ; 1967$.

2 BOCHNER, K.: Am. J. Obst. \& Gynec. 83 : $269 ; 1962$.

3 BRIGGS, R., HERREN, R., THOMPSON, W.: Am. J. Obst. \& Gynec. 84: 436; 1962.

4 BROCHIER, A., and RASCLE, J.: Gynec. et Obst. 65: 67; 1966.

5 CASTELLANOS, R., AGUERO, O., FRANCO, G., y MORENO, L. F.: Rev. Obst. Gin. Venezuela. 29: 65; 1969
6 CLAMAN, A. D., and BELL, M.: Am. J. Obst. \& Gynec. 90: 350; 1964.

7 DOLL, R., HANINGTON, E.: Path. Microbiol. 24: $531 ; 1961$

8 GLICK, J., SALERNO, L., ROYCE, J.: Arch. Gen. Psychiat. 12: 260; 1965.

9 HOFMEISTER, F. J., \& BURGESS, G. F.: Obst. \& Gynec. 6: 162; 1965.

10 ISRAEL, S. L., and WOUTERS, T. B.: Am. J. Obst. \& Gynec. 85: 659; 1963.

11 MARCHETTI, A., and MENAKER, J.: Am. J. Obst. \& Gynec. 59: 1013; 1958.

12 MUSSIO, J. J.: Am. J. Obst. \& Gynec. 4: 443 ; 1962.

13 POLIAKOFF, S. R.: Am. J. Obst. \& Gynec. $76: 746 ; 1958$.

14 SCHAUfFleR, G. C.: "Ginecología Pediátrica". Ed "La Médica". Rosario (R. A.) 1956.

15 SEMMENS, J.: Obst. \& Gynec. 26: 77 ; 1965.

16 SINCLAIR, R.: J. Obstet. and Gyn. Brit. Emp. LIX: 504; 1952. 